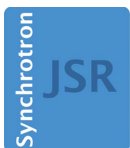

JOURNAL OF SYNCHROTRON RADIATION

ISSN 1600-5775

Received 9 February 2021

Accepted 28 July 2021

Edited by I. Lindau, SLAC/Stanford University, USA

Keywords: white beam; X-ray imaging; micro-CT; real-time imaging.

Supporting information: this article has supporting information at journals.iucr.org/s

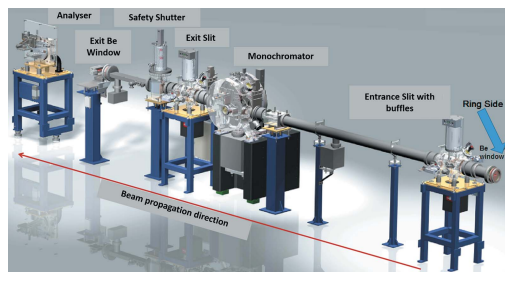

\section{The white beam station at imaging beamline BL-4, Indus-2}

\author{
Ashish K. Agrawal, ${ }^{\mathrm{a} *}$ Balwant Singh,,${ }^{\mathrm{a}, \mathrm{b}}$ Payal Singhai, ${ }^{\mathrm{a}}$ Yogesh Kashyap $^{\mathrm{a}, \mathrm{b}}$ and \\ Mayank Shukla a,b
}

${ }^{\text {a }}$ Technical Physics Division, Bhabha Atomic Research Centre, Trombay, Mumbai 400085, India, and ${ }^{\mathbf{b}}$ Homi Bhabha
National Institute, Anushaktinagar, Mumbai 400094, India. *Correspondence e-mail: ashishka@rrcat.gov.in

The high flux density of synchrotron white beam offers several advantages in $\mathrm{X}$-ray imaging such as higher resolution and signal-to-noise ratio in $3 \mathrm{D} / 4 \mathrm{D}$ micro-tomography, higher frame rate in real-time imaging of transient phenomena, and higher penetration in thick and dense materials especially at higher energies. However, these advantages come with additional challenges to beamline optics, camera and sample due to increased heat load and radiation damage, and to personal safety due to higher radiation dose and ozone gas hazards. In this work, a white beam imaging facility at imaging beamline BL-4, Indus-2, has been developed, while taking care of various instrumental and personal safety challenges. The facility has been tested to achieve $1.5 \mu \mathrm{m}$ spatial resolution, increased penetration depth up to $900 \mu \mathrm{m}$ in steel, and high temporal resolutions of $\sim 10 \mathrm{~ms}$ (region of interest $2048 \times 2048$ pixels) and $70 \mu \mathrm{s}(256 \times$ 2048 pixels). The facility is being used successfully for X-ray imaging, nondestructive testing and dosimetry experiments.

\section{Introduction}

Imaging beamline BL-4 (Indus-2, India) is a national facility for X-ray imaging based research in materials, biomedical, geological and several other domains of science and engineering. It was installed, commissioned and made available to external users in the monochromatic mode in 2014 (Agrawal et al., 2015). Since then, it has been successfully operating with the availability of different micro-imaging techniques, such as propagation-based phase-contrast imaging, micro-tomography, diffraction enhanced imaging, dual-energy imaging etc. (Agrawal et al., 2017; Singh et al., 2017). The facility has been successfully used for numerous applications in a variety of domains in research and industry (Chhajed et al., 2019; Thomas et al., 2019; Choudhary et al., 2018; Agrawal et al., 2019; Mishra et al., 2020; Manzoor et al., 2019; Fatima et al., 2017; Yadav et al., 2018). Monochromatic beam has been used quite successfully with advantages such as availability of diffraction enhanced imaging for high-sensitivity phase contrast, improved edge enhancement in propagation-based phase-contrast imaging, and removal of beam-hardening artefacts in tomography. However, a major part of the incident flux is lost in the process of monochromatization. This substantial reduction in flux leads to longer data acquisition times, reduced signal-to-noise ratios and difficulty in achieving high resolution at BL-4. In several scientific and industrial applications, the demand for higher flux density and increased penetration depth in real-time imaging and in situ material testing experiments far outweighs the advantages offered by a 
monochromatic beam (Patterson et al., 2018; Singh et al., 2014; Yashiro et al., 2017; Reinhart et al., 2014; GarcíaMoreno et al., 2018). In fact, for phase-contrast imaging, there is only a moderate requirement of temporal coherence for many advanced phase contrast techniques, such as X-ray speckle imaging, grating-based phase-contrast imaging etc. (Berujon et al., 2014; Bachche et al., 2017), which is easily fulfilled by broad-spectrum beams provided by synchrotron white beam. Therefore, to overcome current limitations and widen the scope of the beamline in a cost-effective way, it was decided to upgrade the beamline for white beam mode operation.

White beam mode has been implemented at various synchrotron-based imaging beamlines, either in a pure form where spectral distribution is not changed (except for a lower cut using attenuation filters) or as a pink beam with an additional upper cut to remove the higher energy band using a mirror. The installation of white beam mode at any synchrotron beamline depends on its optical design, spectral distribution of the incident beam, heat load etc., and varies in the way by which these challenges are addressed for instrumental and personal safety. Some of the bending magnet beamlines with white beam mode of operation implemented are the Advanced Photon Source (APS), USA [2BM] (Wang et al., 2001), Pohang Light Source, South Korea [5C1] (Jung et al., 2002; Huang et al., 2010), Canadian Light Source, Canada [05B1-1] (Wysokinski et al., 2007), ANKA, Germany [TOPOTOMO] (Rack et al., 2009), APS, USA [7-BM] (Rivers, 2016), Diamond Light Source, UK [B16] (Zhou et al., 2018), Elettra, Italy [SYRMEP] (Sena et al., 2015) etc. These beamlines offer a flux density of $\sim 10^{12}$ photons $\mathrm{s}^{-1} \mathrm{~mm}^{-2}$. Insertion device based beamlines with white beam mode, such as the ID15, ID17 and ID19 beamlines at ESRF (Di Michiel et al., 2005; Rack et al., 2013; Mittone et al., 2020), 32-ID at APS, and JEEP at Diamond Light Source (Tan et al., 2015), facilitate an even higher flux density of $10^{14}-10^{16}$ photons $\mathrm{s}^{-1} \mathrm{~mm}^{-2}$. White beam at BL-4, Indus- 2 , is the synchrotron radiation directly emitted from the bending magnet and received at the experimental station without any major changes in its spectral distribution. It offers orders-of-magnitude higher flux density and wider spectral distribution, and is hence extremely well suited for high-speed X-ray imaging studies of transient phenomena like melting/solidification of materials, the effect of load/environment/chemicals on materials, physiological processes in biological animals and plants (if experimented under controlled dose conditions) etc. The availability of higher flux density also makes it possible to utilize highmagnification camera optics to achieve sub-micrometre resolution in X-ray imaging. The availability of higher energy photons in white beam allows increased penetration depth compared with monochromatic beam, thus allowing the imaging of thicker or denser material.

However, the utilization of white beam presents itself with several instrumental and personal safety challenges such as high heat load, flux density, radiation dose and ozone hazards etc., which at imaging beamline Indus-2 were taken care of successfully through the specific design of optical and mechanical components: a safety system against radiation doses and toxic gases and a new lens-coupled indirect imaging camera. The heat load at the optical components was taken care of by their proper cooling and utilization of attenuation filters. Calculations on spectral flux density modification by different attenuation filters were carried out. Installation of a noble gas $(\mathrm{He})$ flow chamber ensured the protection of optical components exposed to air against ozone hazards. Installation of ozone monitors, a ventilation system and access control with suitable safety interlocks was ensured for personal safety against ozone toxicity. Moreover, the white beam, which is rich in high-energy X-rays, also undergoes scattering (elastic and Compton), thereby contributing to increased scattered radiation dose along with noise (bright pixels) in the imaging detector. A specific lens-coupled indirect mode imaging with microscope optics was used for high-resolution, high-framerate imaging using the white beam. The dose rate of white beam is also higher compared with monochromatic beam, thus controlled exposure using a fast shutter and online dose monitoring using a calibrated ion chamber were ensured for samples which are dose sensitive, especially soft materials and biological tissues. The shielding walls and doors of the hutches are designed to ensure adequate radiation safety to the personnel in the control room against increased synchrotron and Bremsstrahlung radiation. Additional safety interlocks were also installed with radiation monitors, a ventilation system, door locks and a beam shutter. In this paper, we discuss different features of these systems to upgrade the imaging beamline in white beam operation mode. Experimental results of $2 \mathrm{D}, 3 \mathrm{D}$ and real-time imaging techniques on various representative samples are presented to demonstrate the successful implementation of this facility.

\section{Technical details}

The Indus-2 imaging beamline at the Indian synchrotron source (Deb et al., 2013) is installed at a $10^{\circ}$ port of a bending magnet source of strength $1.5 \mathrm{~T}$ at $\mathrm{BL}-4$ with horizontal acceptance of $5.5 \mathrm{mrad}$ and vertical acceptance of $0.5 \mathrm{mrad}$ (Agrawal et al., 2015). The characteristics of the beamline are given in Table 1. A schematic of the beamline showing major components is shown in Fig. 1. The first component of the beamline, i.e. the beryllium $(\mathrm{Be})$ window, is at $18.5 \mathrm{~m}$ from the tangent point to filter the lower energy spectrum of the incoming beam. The transmitted beam can be cropped using a pair of entrance slits with baffles $(19.5 \mathrm{~m})$. The cropped beam can be passed through a double-crystal monochromator (DCM) which, if no monochromatization is required for white beam operation, has provision to remove the first crystal from the beam path thus allowing passage of the incident beam without any spectral modification. This beam passes through a second Be window (exit Be window) and reaches the experimental station where it can be used for various experiments. The spectral distributions of this beam emitted directly from the bending magnet source and after passing through two Be windows of thickness $0.4 \mathrm{~mm}$ each are shown in Fig. 2(a). 


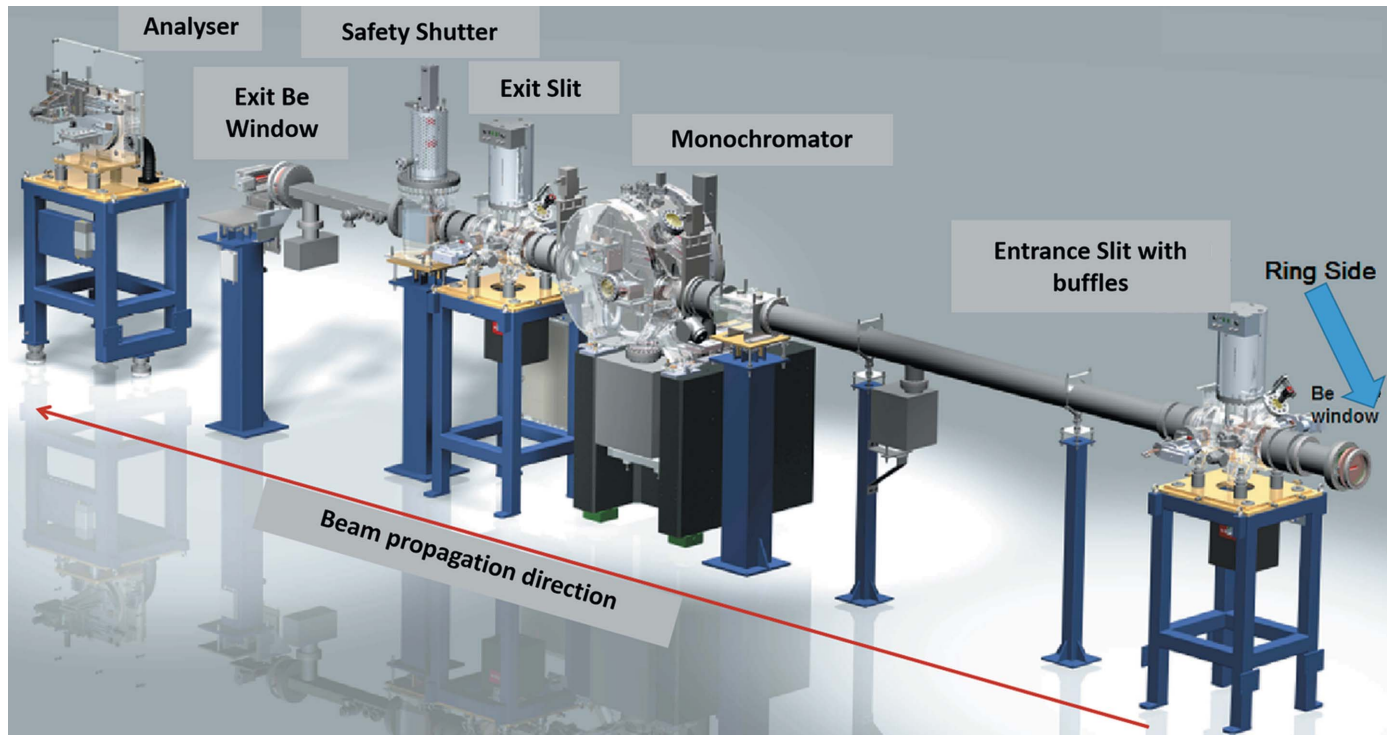

Figure 1

Schematic of the imaging beamline (Bl-4) at Indus-2 showing major components and their sequence.

Table 1

Design parameters and beam characteristics at the imaging beamline.

\begin{tabular}{ll}
\hline Parameter & Typical value \\
\hline Ring energy $(\mathrm{GeV})$ & 2.5 \\
Ring current $(\mathrm{mA})$ & 200 \\
Source & Bending magnet, 1.5 T dipole \\
Operational modes & White and monochromatic \\
Monochromator & $\mathrm{Si}(111) \mathrm{DCM}$ \\
Energy range $(\mathrm{keV})$ & $8-35$ \\
Energy resolution & $3.86 \times 10^{-4}$ at $12 \mathrm{keV}$ in monochromatic mode \\
Acceptance (mrad) & $5.5(\mathrm{H}) \times 0.5(\mathrm{~V})$ \\
Photon flux & $\sim 1.74 \times 10^{8}$ photons s ${ }^{-1} \mathrm{~mm}^{-2}(120 \mathrm{~mA})^{-1}$ at \\
& $12 \mathrm{keV}$ \\
& $($ monochromatic) \\
& $\sim 10^{16}$ photons s ${ }^{-1}$ at $2.5 \mathrm{GeV}$ and $300 \mathrm{~mA}$ \\
Sample stage & Five-axis $(2 \mathrm{D}$ translation + rotation $+2 \mathrm{D}$ tilt $)$ \\
Detectors & $\mathrm{CCD}$, flat panel, X-ray microscope, sCMOS camera \\
Detector stage & Three-axis manipulator for detectors $(X Y Z)$ \\
\hline
\end{tabular}

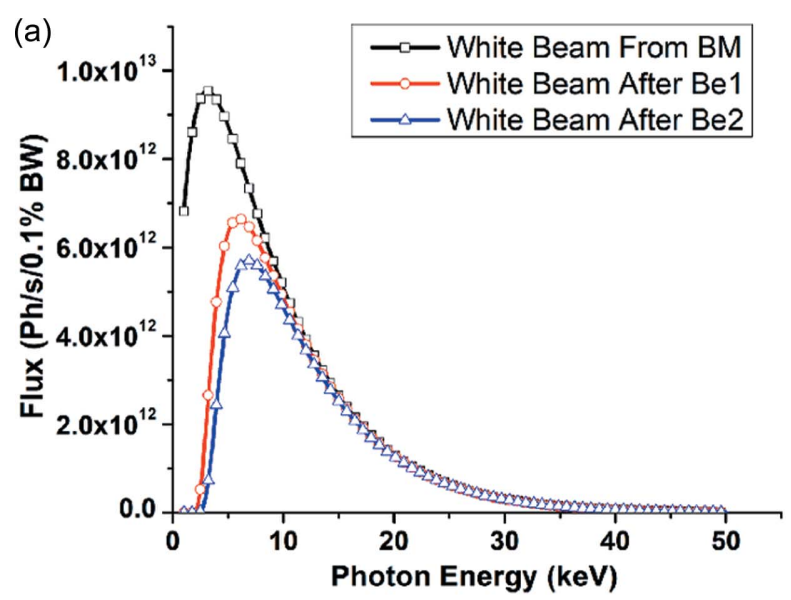

\section{Instrumentation}

\subsection{White beam operation}

Provision of easy switching between monochromatic and white beam operation is facilitated at the imaging beamline through the removal of the $\mathrm{Si}(111)$ first crystal in the DCM thus allowing passage of the white beam without any spectral modification. Further optical instruments such as analyzer crystals for diffraction enhanced imaging and ion chamber are also removed from the beam path to avoid their potential damage due to white beam exposure. A suitable interlock is provided to ensure their off position. The hutch ventilation, radiation monitors, water flow signals, vacuum, ozone monitors etc. are also interlocked with hutch doors and beam opening shutter to ensure personal and instrumental safety during white beam operation. A schematic of the logical interfacing of various beamline equipment and personal safety

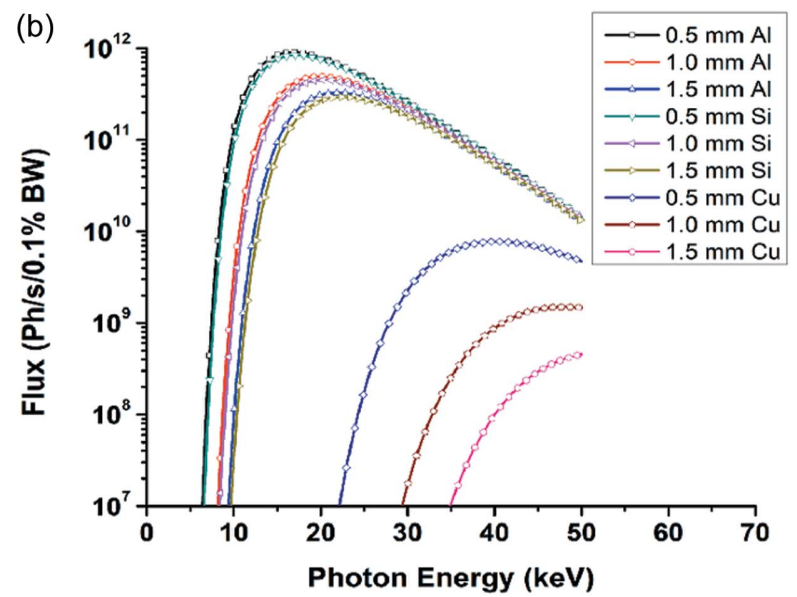

Figure 2

(a) Spectral distribution of the white synchrotron beam from the bending magnet (BM) source before and after the Be windows. (b) Spectral distribution of the beam considering different thicknesses of $\mathrm{Al}, \mathrm{Si}$ and $\mathrm{Cu}$ filters. 


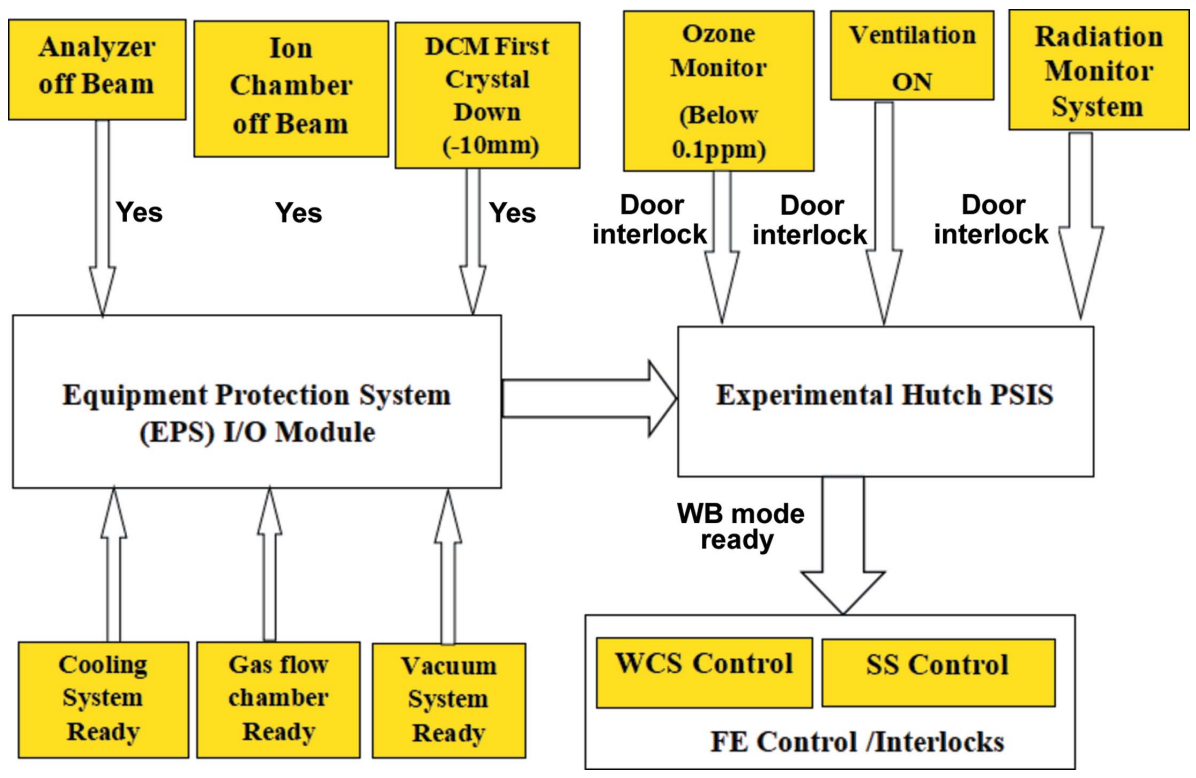

Figure 3

Schematic of the logical interfacing of instruments at the imaging beamline for switching to white beam mode operation.

Table 2

Parameters of the synchrotron used in the calculations on attenuation filters.

\begin{tabular}{ll}
\hline Parameter & Value at Indus-2 \\
\hline Magnetic radius, $\rho(\mathrm{m})$ & 5.56 \\
Magnetic field, $B_{\mathrm{o}}(\mathrm{T})$ & 1.5 \\
$E /\left(m c^{2}\right), \gamma$ & 4892.37 \\
Beam energy, $E(\mathrm{GeV})$ & 2.5 \\
Beam current, $I(\mathrm{~A})$ & 0.200 \\
Critical energy, $E(\mathrm{eV})$ & 6234.6162 \\
\hline
\end{tabular}

system is shown in Fig. 3, which helps in easy and safe operation of the beamline in white beam mode. After all these provisions are completed, white beam is permitted in the experimental station.

\subsection{X-ray attenuators}

Attenuation filters are necessary to optimize the flux density of the incident beam at the sample and detector position to avoid their damage or saturation of the CCD detector in the imaging camera (Davison et al., 2017). Apart from flux optimization, filters preferentially attenuate the lower energies from the incident beam making its spectrum harder by shifting the mean energy towards the higher side. This is useful for improving the signal-to-noise ratio (SNR) in the imaging of thick or more attenuating samples and reducing beam-hardening artefacts (Rana et al., 2015). The utilization of filters also reduces toxic gas hazards in the hutch by reducing the flux density of the incident beam leading to significantly fewer events of ozone formation. Attenuation filters also help to optimize the radiation dose to the sample in biomedical imaging (Ay et al., 2013). If a metallic filter with high thermal conductivity is used with suitable cooling, it also helps in reducing the heat load of the beam to the beamline passing through the first and second Be windows. This spectrum is used as an input beam for comparing modifications in the spectral distribution and flux density of the beam after passing though different filters of varying thicknesses. Potential filter materials are those with high thermal conductivity, high melting point, high mechanical strength, easy machining, no toxicity, no internal structural defects etc. These properties are mostly fulfilled by metals or diamond. Indus- 2 is a medium-energy ring $(2.5 \mathrm{GeV})$; therefore the white beam spectrum does not have a significant contribution from energies higher than $50 \mathrm{keV}$. The photon flux density at an energy of $50 \mathrm{keV}$ is $\sim 10^{10}$ photons $\mathrm{s}^{-1}(0.1 \% \text { bandwidth })^{-1}$; therefore, filters of very high atomic numbers have not been used. Thus, potential filter materials include Be, B, C, polymers, $\mathrm{Al}, \mathrm{Si}, \mathrm{Cu}$ etc. Polymer filters were not used to avoid their distortion due to the heat load of the X-ray beam, difficult machining and the possibility of internal structure. Beryllium is already installed at the beamline for vacuum isolation purposes and the slight increase in thickness does not alter the spectrum much. Boron was not used due to its toxicity and diamond could not be used due to its high cost. The modification of the white beam spectrum at the experimental station after filtering through different thicknesses of the remaining filter materials such as $\mathrm{Al}$ (density $2.7 \mathrm{~g} \mathrm{~cm}^{-3}$ ), $\mathrm{Si}$ $\left(2.33 \mathrm{~g} \mathrm{~cm}^{-3}\right)$ and $\mathrm{Cu}\left(8.98 \mathrm{~g} \mathrm{~cm}^{-3}\right)$ is illustrated in Fig. $2(b)$.

The outgoing white beam has different lower-energy cutoffs depending on the material and thickness of the filters. The mean energy of the spectral distribution increases with the thickness of the filters leading to beam hardening. The flux density at all energies is also reduced to some extent depending on the thicknesses of the filters. The $\mathrm{Al}$ and $\mathrm{Si}$ moderately reduce the flux density and energy distribution whereas $\mathrm{Cu}$ does this significantly. The changes in spectral distribution and flux density due to different thicknesses of 
various filters in these figures serve as a ready reference during white beam experiments (phase-contrast imaging, real-time imaging and in situ tomography etc.) for acquisition time, penetration depth and beam-hardening artefact optimization.

\subsection{Radiation safety system}

White beam operation is carried out at a significantly higher beam flux density $\left(\sim 10^{12}\right.$ photons $\mathrm{s}^{-1} \mathrm{~mm}^{-2}$ compared with $10^{9}$ photons s $\mathrm{s}^{-1} \mathrm{~mm}^{-2}$ in the monochromatic beam) and wider spectral distribution with higher-energy photons up to several hundreds of $\mathrm{keV}$. Apart from synchrotron radiation, the beamline also receives a significantly higher amount of Bremsstrahlung radiation from the ring. Both of these radiations are also scattered by different beamline components and this leads to a higher radiation dose to the hutches. This poses the requirement of increased radiation safety against dose inside the experimental hutch in white beam operation mode. At the imaging beamline, the hutch walls and doors are made up of two layers of $1.4 \mathrm{~mm}$-thick stainless steel (SS) sheets and between them is sandwiched a layer of $3 \mathrm{~mm}$-thick lead. The roof is made of $3 \mathrm{~mm}$-thick SS. The doors of the hutches are interlocked with a beam opening shutter and radiation monitors. The radiation monitors are installed in the hutches to continuously measure radiation dose and display it in the control area. Radiation dosimeters are also interlocked with the beam shutter installed in the experimental station which is closed immediately as soon as the higher dose is observed in the control room during any accidental scenario. The radiation safety system is periodically tested to ensure its functionality. In order to control the radiation exposure to experimental samples that are sensitive to radiation dose, a fast shutter system made up of OFHC (oxygen-free high thermal conductivity) copper is used to provide conditioned X-ray radiation to the sample. The rotary shutter is equipped with a stepper motor (500 steps per revolution) allowing acceleration of 900000 steps s$^{-1}$. The shutter has an aperture $140 \mathrm{~mm}$ (W) $\times 15 \mathrm{~mm}(\mathrm{H})$ in size with a typical operating and closing time of $<20 \mathrm{~ms}$. Apart from the fast shutter, an ion chamber is also installed, tested and calibrated for online measurement of the intensity of the beam and radiation dose to the samples during experimental studies on biological samples.

\subsection{Beamline exit window and ozone safety system}

White beam, as it passes through the air in the experimental station, generates a significant concentration of ozone depending on the stored beam current in the accelerator ring (Table 3). Ozone causes oxidation-induced corrosion damage to the instruments in the experimental stations, especially Be windows and the reflective coating on the mirror of the lenscoupled imaging detector. The exit Be window of the imaging beamline is exposed to air, and a prolonged white beam operation may cause significant damage by creating small pits through oxidation (Yang et al., 1993). Similarly the Al coating of the mirror in the lens-coupled imaging detector may also be oxidized and may lead to artefacts in the images. To avoid this, ozone generation should be minimized in the vicinity of the $\mathrm{Be}$
Table 3

Ozone measurement at various beam currents and ventilation conditions.

\begin{tabular}{lllll}
\hline Location & $\begin{array}{l}\text { Beam } \\
\text { current } \\
\text { (mA) }\end{array}$ & Ventilation & $\begin{array}{l}\text { Ozone } \\
\text { (p.p.m.) }\end{array}$ & $\begin{array}{l}\text { Time to } \\
\text { background } \\
\text { (s) }\end{array}$ \\
\hline Near exit Be window & $\sim 54$ & Off & $\sim 3.3$ & $\sim 120$ \\
& & On & $\sim 0.3$ & $\sim 30$ \\
Near exit Be window & $\sim 118$ & Off & $\sim 7.2$ & $\sim 240$ \\
& & On & $\sim 0.8$ & $\sim 60$ \\
Sample position & $\sim 115$ & Off & $\sim 0.4$ & $\sim 40$ \\
& & On & $\sim 0.2$ & $\sim 20$ \\
\hline
\end{tabular}

window and Al-coated mirror through the creation of a rough vacuum in a chamber next to it or with continuous flow of a noble gas to reduce the ozone density.

We have designed, fabricated, installed and tested a dualpurpose chamber with a non-metal (Kapton) window which can be configured to create a rough vacuum $\left(\sim 10^{-3} \mathrm{mbar}\right)$ or used to flush out the generated ozone using flow of a suitable noble gas such as $\mathrm{He}$ (Fig. 4). The chamber has a pair of Kapton windows $150 \mathrm{~mm} \times 15 \mathrm{~mm}$ in size. They are designed for passage of monochromatic as well as white beam to enable easy switching between both modes. The Kapton windows inside can be easily installed and replaced. The chamber also allows the installation of various filters to optimize radiation dose, spectral distribution, heat load and flux of the white beam as discussed in the previous section. The utilization of these filters also significantly reduces the risk of Kapton window damage under heat load.

Ozone also poses a safety risk to personnel due to its toxic nature. When inhaled, it can damage the lungs or may cause chest pain, coughing, shortness of breath and throat irritation
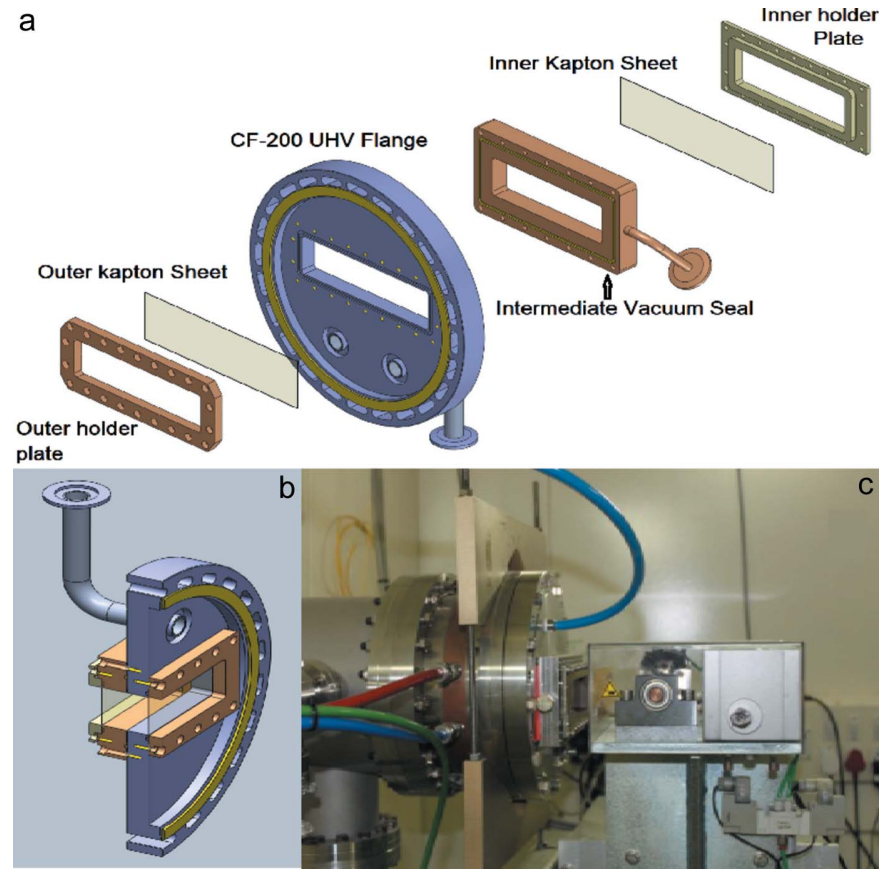

Figure 4

(a) Components of the dual-purpose vacuum/gas flow chamber. (b) The assembled system. (c) The installed system at the beamline. 
(Cook \& Nakano, 2011). The concentration of ozone produced in the experimental station at different stored beam currents of Indus-2 was measured and found to be above acceptable safety criteria (0.1 p.p.m.). We have ensured monitoring, ventilation and access control of the experimental station with safety interlocks to avoid any personal hazard due to ozone. An ozone monitor is installed near the experimental table to measure and record the ozone concentration in real time. The safe threshold of this monitor is interlocked with the hutch door to avoid accidental access to the experimental station until the ozone level is low. The hutch is equipped with exhaust fans for ventilating the area. The hutch doors are also interlocked with a beam access shutter to restrict hutch access until an additional delay time is passed (currently set at $5 \mathrm{~min}$ ). The effectiveness of ventilation and other provisions to reduce ozone concentration up to the background level was measured quantitatively (Table 3).

\subsection{X-ray microscope}

The area detector used in white beam imaging faces several challenges such as high heat load, excess flux, damage of optics due to radiation dose and oxidizing gases. Ionization due to the X-ray beam and its high heat load may potentially damage camera optics, electronics and the CCD itself. Though attenuating filters are used to optimize the incident beam flux, the camera is also specially designed to avoid any damage to electronics and optics (Lai et al., 1995; Douissard et al., 2012; Rack et al., 2010). We have installed an indirect mode highdose camera specific to white beam operation at the imaging beamline. The camera has a CCD or sCMOS detector and optics mounted at $90^{\circ}$ from the incident beam (Fig. 5). The scintillator support holds the crystal scintillator, such as
GGAG(Ce) (in-house developed), $\operatorname{LuAg}(\mathrm{Ce})$ or YAG(Ce) (Crytur, Czech Republic), to convert the incident X-ray beam into optical signals which are reflected towards the optics and camera at $90^{\circ}$ using an aluminium-coated silicon mirror (Optique Peter, France). Long-working-distance objective lenses (Mitutoyo, Japan) are installed to focus the optical beam to the camera depending on its numerical aperture and magnification. Objective lenses are protected from Comptonscattered radiation-induced radiation damage using titanium shielding. The high flux density of the X-ray beam causes radiation damage in the scintillator; thus they need to be changed frequently. Ozone produced in the camera may cause oxidation of the aluminium coating in the mirror; therefore, a provision for continuous flow of noble gas is provided in the space near the mirror.

The advantage of white beam imaging relates to its high flux density which allows real-time imaging and in situ studies. Therefore, the imaging sensor used should have a high frame rate for fast imaging. In our camera, we can alternatively use PCO-2000 CCD and PCO-Edge sCMOS detectors (PCO-AG Germany) which have distinct features in terms of imaging frame rate, resolution, electronic noise etc. The PCO-Edge

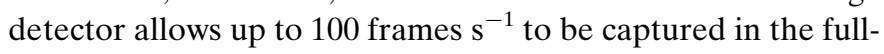
frame of $2048 \times 2048$ pixels and a higher frame rate if used with binning or a smaller region of interest (ROI) (up to 1400 frames s $^{-1}$ at ROI $\left.2048 \times 256\right)$. Radiographic images of the JIMA pattern (JIMA, Japan) with two different optical magnifications $(5 \times$ and $10 \times)$ are shown in Fig. 6 for comparison of the highest resolution achieved (see also Table 4). In Fig. 7, the dependence of SNR on image acquisition time for $100 \mathrm{~ms}$ [Figs. 7(a) and 7(b)], $200 \mathrm{~ms}$ [Figs. 7(c) and $7(d)$ ] and $400 \mathrm{~ms}$ [Figs. 7(e) and 7(f)] and its effect on the resolution are compared.
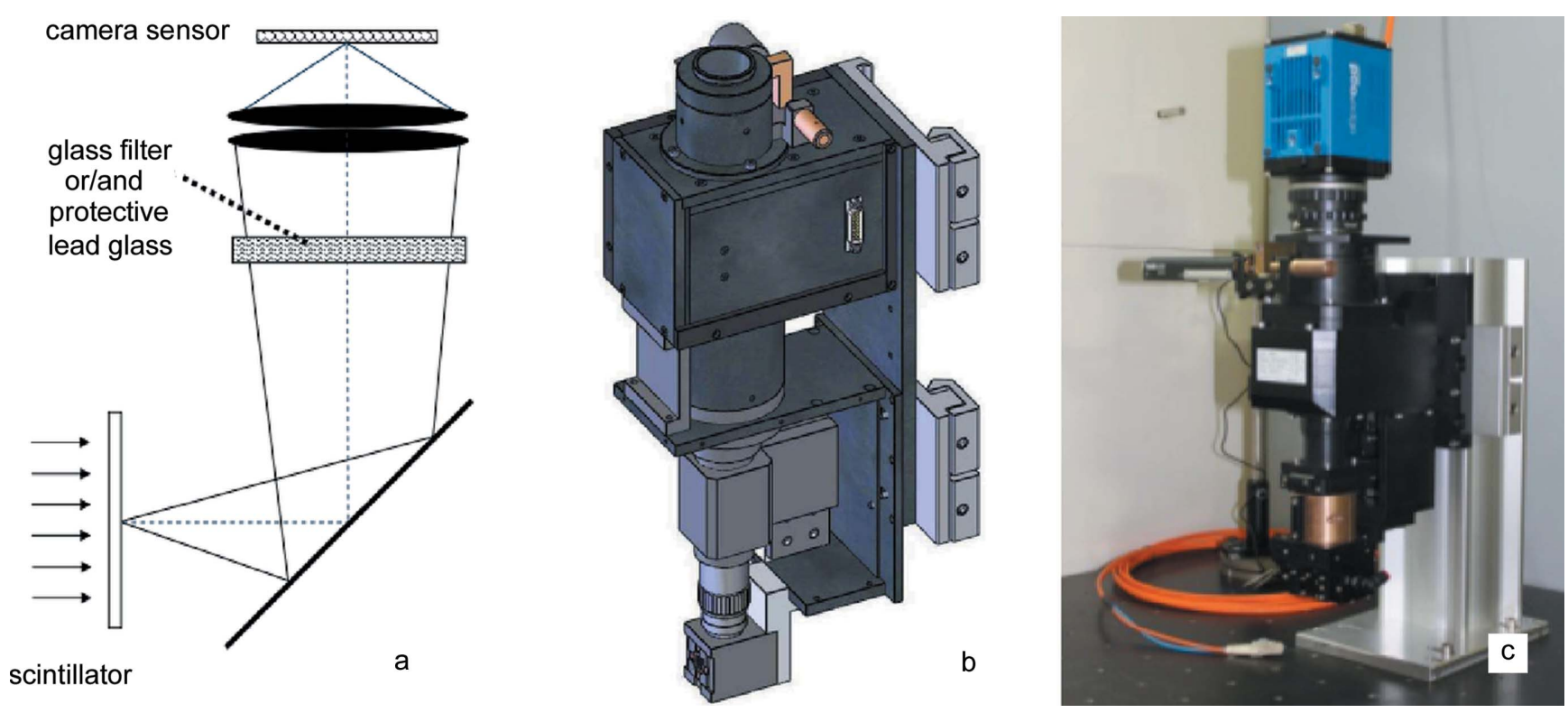

Figure 5

(a) Design (Rack et al., 2010) and (b) 3D model (Douissard et al., 2012) of the white beam imaging lens-coupled camera. (c) The installed system at the imaging beamline. 

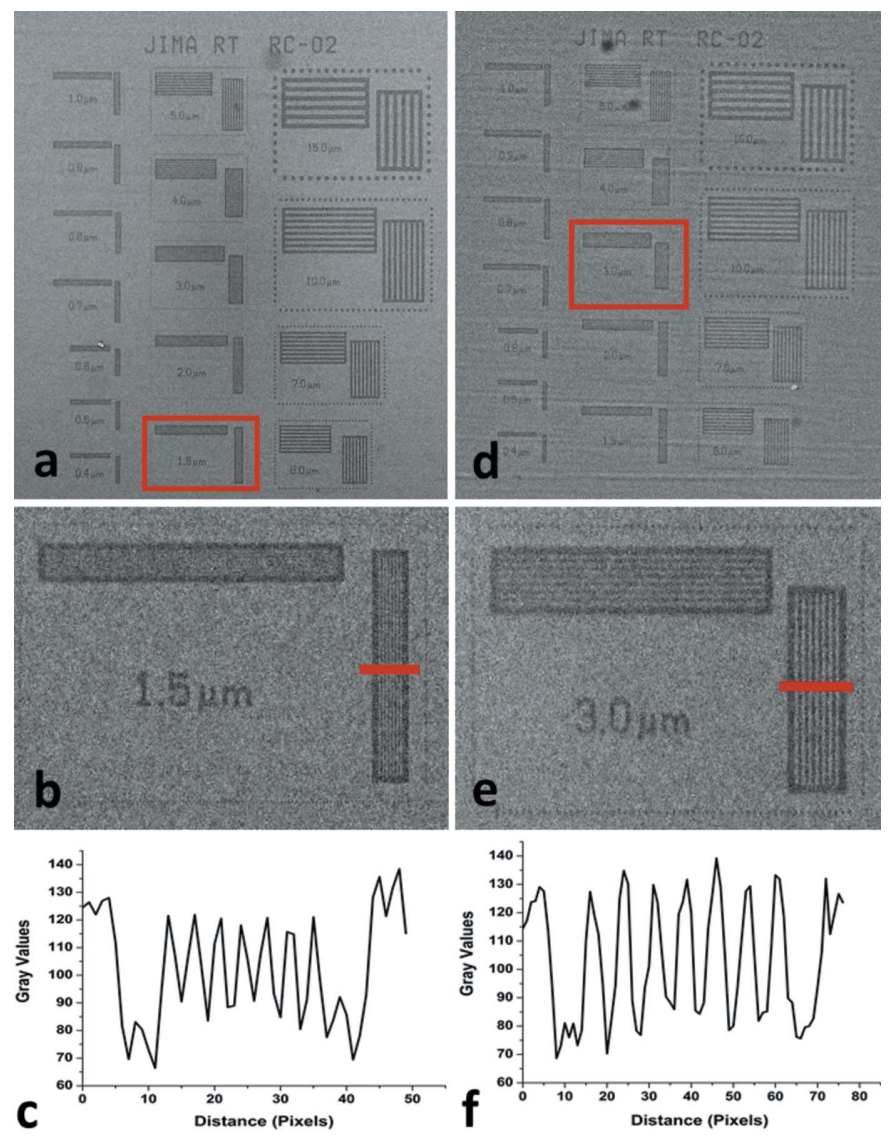

Figure 6

Radiographic images of the JIMA pattern with two different optical magnifications $[10 \times(a-c)$ and $5 \times(d-f)]$ for comparison. Regions of $1.5 \mu \mathrm{m}$ lines in the $10 \times$ objective and $3.0 \mu \mathrm{m}$ lines in the $5 \times$ objective are highlighted along with their respective line profiles.
Table 4

Experimental parameters of the white beam imaging detector at different objective magnifications.

\begin{tabular}{llllll}
\hline $\begin{array}{l}\text { Objective } \\
\text { magnification }\end{array}$ & NA & $\begin{array}{l}\text { Effective } \\
\text { pixel size }\end{array}$ & $\begin{array}{l}\text { Resolution } \\
(\mu \mathrm{m})\end{array}$ & $\begin{array}{l}\text { Field of view } \\
(\mathrm{mm})\end{array}$ & $\begin{array}{l}\text { Typical } \\
\text { acquisition } \\
\text { time }(\mathrm{ms})\end{array}$ \\
\hline $2 \times$ & 0.055 & 3.7 & $\sim 7$ & 7.5 & 200 \\
$5 \times$ & 0.140 & 1.48 & $\sim 3$ & 3.0 & 400 \\
$10 \times$ & 0.280 & 0.74 & $\sim 1.5$ & 1.5 & 800 \\
\hline
\end{tabular}

\section{Applications}

\subsection{High-energy white beam imaging}

In order to demonstrate the higher penetration capability of the white beam, we have used two SS rods of $5.0 \mathrm{~mm}$ diameter. Each of these rods was placed one by one within a hollow cylindrical SS tube of diameter $6.6 \mathrm{~mm}$ and wall thickness $450 \mu \mathrm{m}$ leaving an annular space of $350 \mu \mathrm{m}$. These rods had a notched space of length $5 \mathrm{~mm}$ and depth $1.5 \mathrm{~mm}$ which were filled with glass and epoxy resin, respectively. A schematic of the tube with an annulus and notches is shown in Fig. 8(a). Radiographic images of the rods with the resin and glass fillings acquired using white beam $(1.0 \mathrm{~mm} \mathrm{Cu}$ filter and $1.5 \mathrm{~mm}$ $\mathrm{Al}$ filter) are shown in Figs. 8(b) and 8(c), respectively. In this experiment, the beam needs to penetrate $900 \mu \mathrm{m}$ of SS to image the notch in the inner SS rod. A special annular enclosure of the tube was used to avoid saturation of the detector directly exposed by the incident X-ray beam. The images were acquired for an acquisition time of $30 \mathrm{~s}$ using the PCO-2000 camera in the white beam microscope with a $100 \mu \mathrm{m}$-thick $\mathrm{LuAg}(\mathrm{Ce})$ scintillator and $5 \times$ optical magnifications. The acquired images were band-pass filtered using
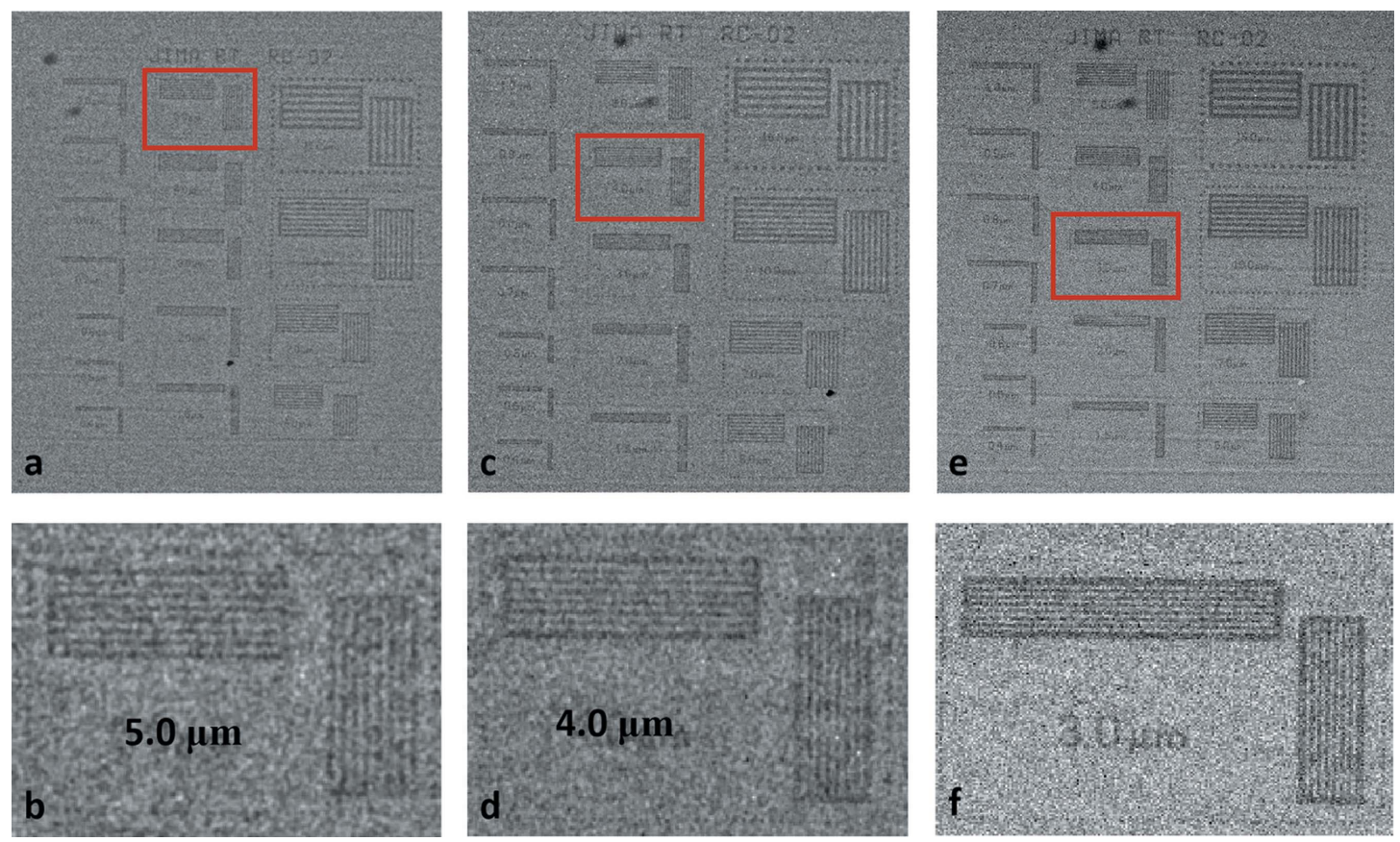

Figure 7

Radiographic images of the JIMA pattern at 5× magnification for different data acquisition times $100 \mathrm{~ms}(a, b), 200 \mathrm{~ms}(c, d)$, and $400 \mathrm{~ms}(e, f)$. The highlighted regions show the effect of acquisition time on SNR and resolution (SNR values for these acquisition times are $120 \mathrm{~ms}, 300 \mathrm{~ms}$ and $500 \mathrm{~ms}$, respectively). 

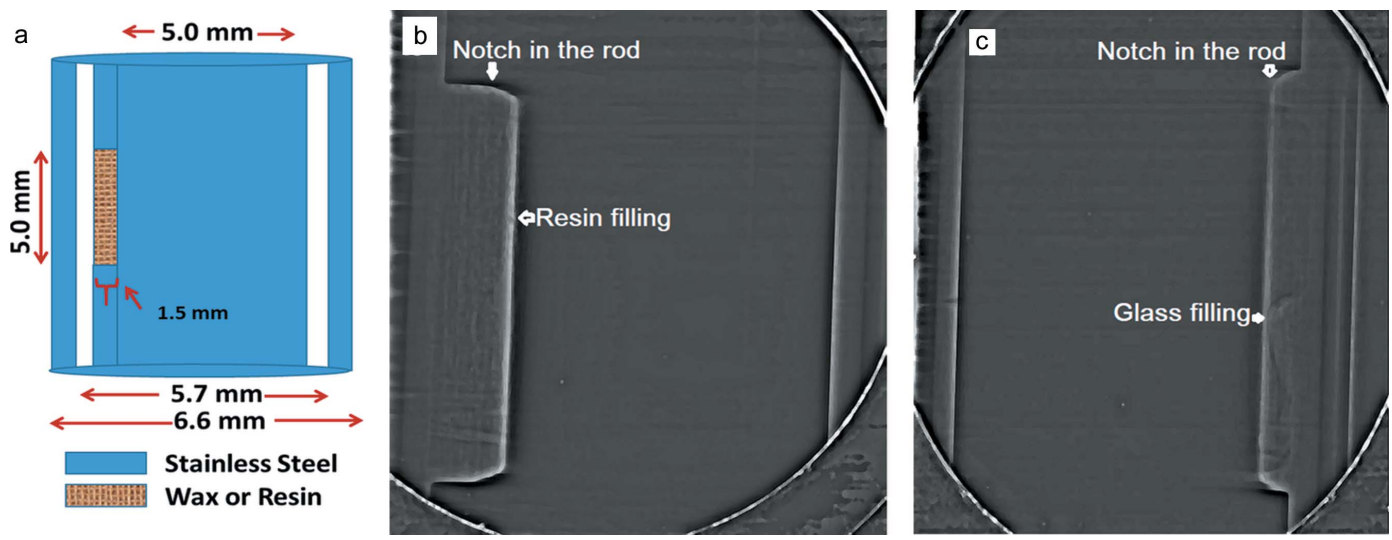

Figure 8

(a) Schematic of a hollow SS tube sample with inserted notched rod. Radiographic images of $(b)$ epoxy resin filling and $(c)$ glass filling inside the notch of the SS rod.

fast Fourier transform (FFT) to reduce speckle noise and enhance the contrast of features in the notch. The epoxy resin and glass filling can be clearly seen in the images with distinguishable edges. The contrast at the edge is also sufficient to identify the features after penetration through the rod. This experiment confirms the capability of white beam for imaging soft materials like resin and glass embedded deep in highly absorbing materials such as stainless steel.

\subsection{High-resolution phase-contrast imaging}

On account of the availability of higher flux density in white beam mode, the acquisition time for radiographic images is reduced substantially. Since the flux density is three to four orders of magnitude higher compared with monochromatic mode with a $\mathrm{Si}(111) \mathrm{DCM}$, the acquisition time is reduced from several seconds in monochromatic mode to around $100 \mathrm{~ms}$ in white beam mode without sacrificing the contrast and SNR in the acquired images. In Fig. 9, we have compared the radiographic projection images of TRISO (Tri-Iso coated) fuel particles using a monochromatic beam of $16 \mathrm{keV}$ and a white beam with a $1.5 \mathrm{~mm} \mathrm{Al} \mathrm{filter.} \mathrm{The} \mathrm{sample} \mathrm{was} \mathrm{kept} 26 \mathrm{~m}$
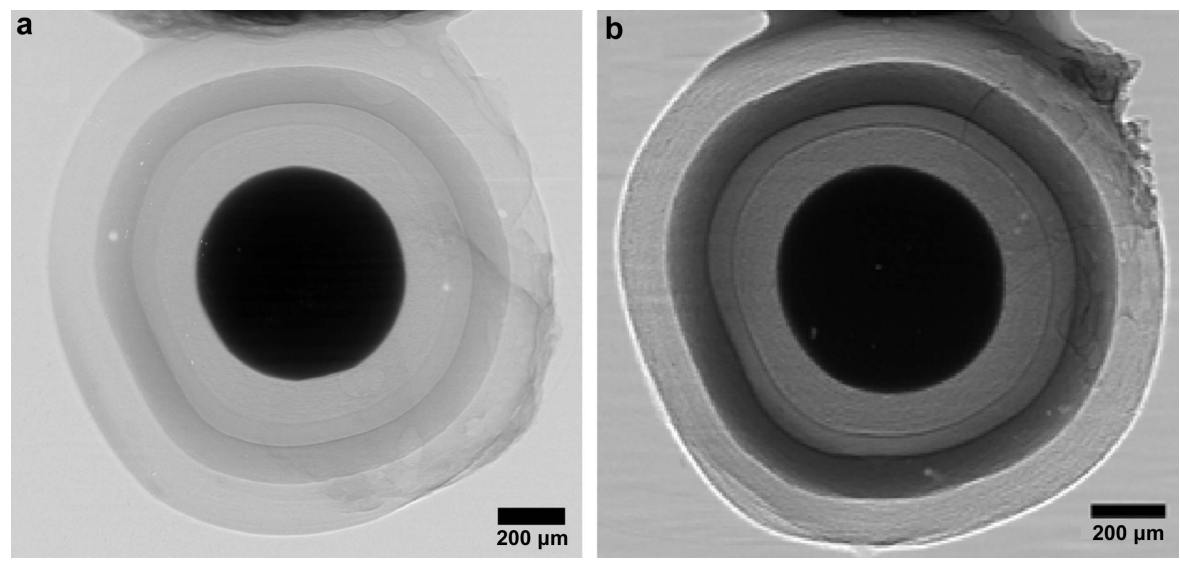

Figure 9

High-resolution phase-contrast radiographic images of a TRISO (Tri-Iso) coated $\mathrm{ZrO}_{2}$ particle using $(a)$ white and $(b)$ monochromatic beam. from the source whereas the sample-to-detector distance was $150 \mathrm{~mm}$ for monochromatic and white beam images. Both images show phase-contrast enhancement in the different layers of pyrolitic graphites and $\mathrm{SiC}$ along with fine microstructural details of the particle. Although the phase contrast is relatively better in a monochromatic beam, the white beam image also shows sufficient phase contrast to visualize all the layers very well while offering the advantage of lower acquisition time against the $16 \mathrm{keV}$ monochromatic beam $(300 \mathrm{~ms}$ compared with $300 \mathrm{~s}$ ). This advantage of white beam offers the possibility of in situ phase contrast tomography experiments on these samples.

\subsection{Real-time imaging}

With white beam, high-resolution imaging at reduced acquisition time and improved SNR leads us to the opportunity of real-time imaging making it possible to observe timedependent phenomena in real time. Some examples of these kinds of experiments include in situ visualization of cavity formation and inter-layer bonding in additive manufacturing, melting and solidification of metals and alloys, in situ effects of different mechanical loads on materials, live imaging of physiological functioning of organs in small animals, dissolution of pharmaceutical tablets and capsules etc. These, however, require capture and transfer of images at a sufficiently high frequency using a suitable high-frame-rate camera. We have used our white beam module and PCO-Edge camera for real-time imaging experiments on a pharmaceutical tablet to study its time-dependent dissolution in water and growth of air bubbles in its matrix with time (Fig. 10). A video file is included in the supporting information showing this phenomenom. We have achieved the highest frame rate of 100 frames s$^{-1}$ for full-frame. The effect 
of ROI selection was also tested, which has a significant impact on the frame capturing frequency, and we have tested up to 1400 frames s$^{-1}$ with an ROI of $256 \times 2048$ using a PCO-Edge camera. Higher-speed imaging would be possible with the availability of a higher-speed sCMOS camera.
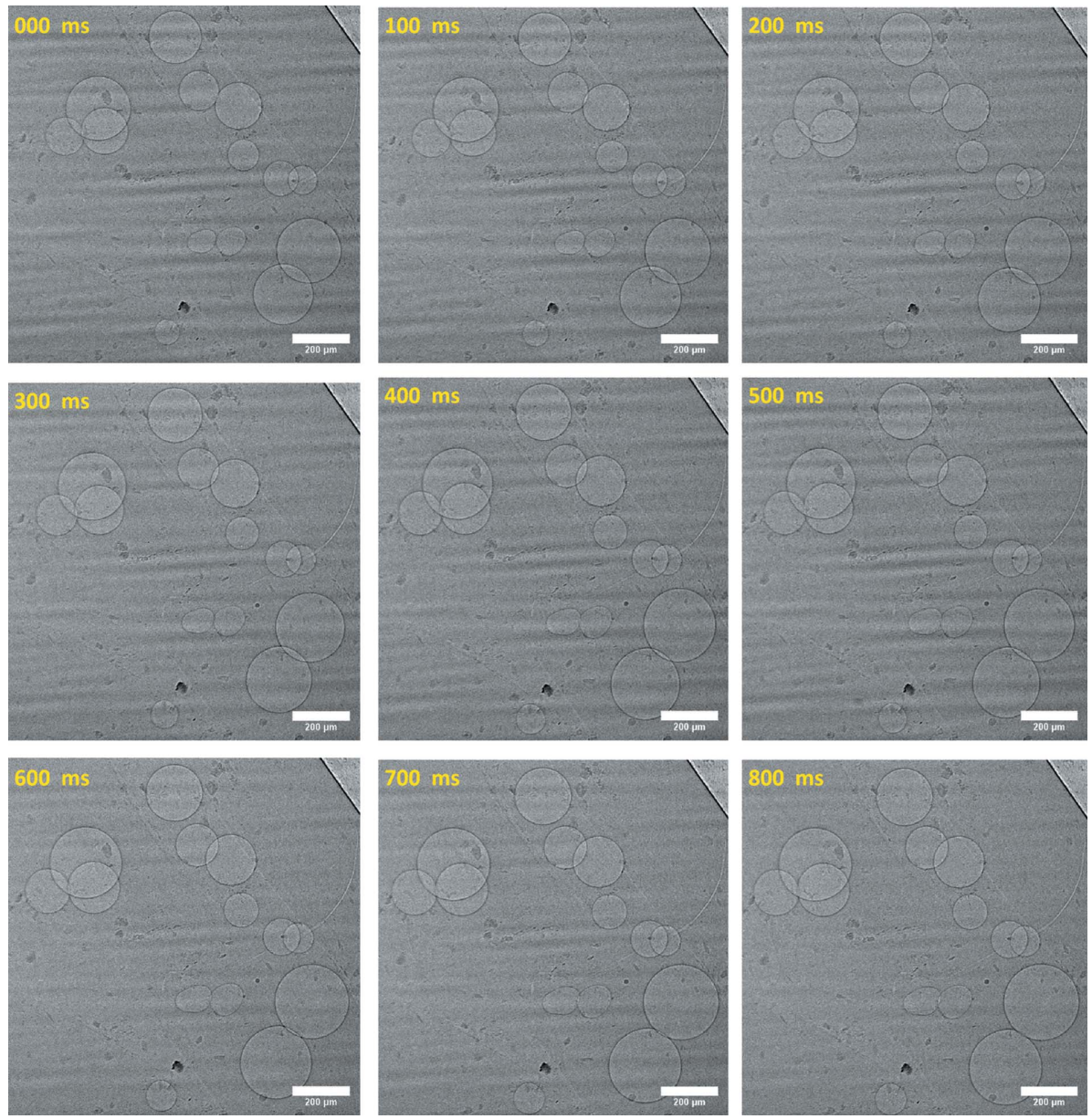

Figure 10

Fast radiographic microscopy of the dissolution of a pharmaceutical tablet in water and growth of air bubbles in its matrix with time (scale bar is $200 \mu \mathrm{m}$ in all images).

\subsection{High-resolution micro-CT imaging}

For testing the high-resolution 3D imaging capabilities of white beam CT, we characterized an AZ80 magnesium alloy sample of dimensions $1 \mathrm{~mm} \times 1 \mathrm{~mm} \times 5 \mathrm{~mm}$ at $5 \times$ magnification which provides a resolution of $3 \mu \mathrm{m}$. The micro-CT data collection was completed in $8 \mathrm{~min}$ with acquisition of each projection in $200 \mathrm{~ms}$ for stepwise rotation of the sample using a stepper motor interfaced with a CCD camera. The resulting reconstructed slice image is shown in Fig. 11. The Al-Mn particles embedded in the $\mathrm{Mg}$ matrix (size range $5-10 \mu \mathrm{m}$ ) can be clearly seen; however, $\mathrm{Mg}-\mathrm{Al}$ particles are not observed due to the lack of sufficient contrast. The voids in the sample can also be seen clearly. A 3D image of the sample with distributed Al-Mn particles and voids is also shown in Fig. 11(c).

\section{Conclusions}

The white beam facility at imaging beamline Indus-2 has been designed, developed, installed and commissioned successfully by solving various challenges relating to higher heat load, flux density, radiation dose and ozone. The specific design of optical and mechanical components, a new lenscoupled indirect imaging camera, and a safety system against radiation doses and toxic gases allow safe operation of the beamline. Experimental results related to improved penetration, achievement of high resolution, high SNR, reduced exposure time, high
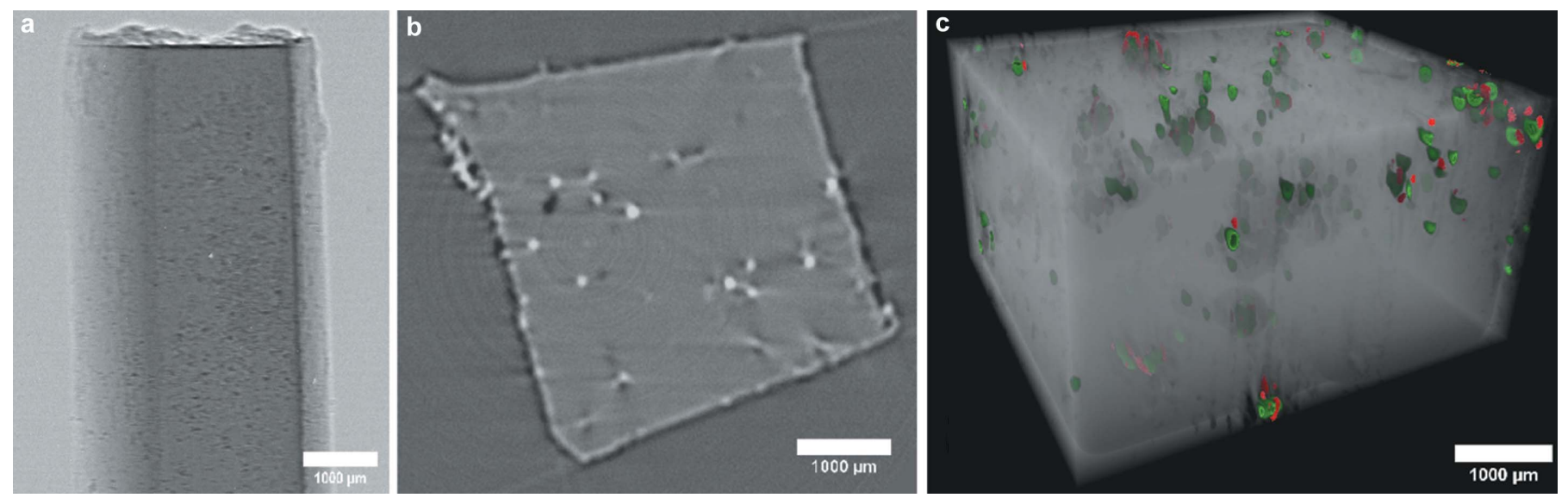

\section{Figure 11}

High-resolution tomographic 3D imaging of an AZ80 magnesium alloy sample. (a) Flat-field-corrected radiographic projection. (b) Tomographic slice image showing voids and Al-Mn particles embedded in an Mg matrix. (c) 3D distribution of Al-Mn particles (green) and voids (red). 
frame rate and 3D imaging are presented to demonstrate the capabilities of white beam based X-ray imaging and successful implementation of this facility. Future efforts are intended to extend the capabilities of the facility for in situ imaging of materials under different mechanical and thermal load and in vivo imaging applications in biomedical imaging.

\section{Acknowledgements}

The author acknowledges Dr S. M. Yusuf, Director Physics Group, and Dr T. V. C. Rao, Head Technical Physics Division, for their support and encouragement in beamline development and utilization activity at the imaging beamline. The authors also acknowledge the support of Dr S. S. Singh, I. I. T. Kanpur and colleagues from IGCAR Kalpakkam for providing test samples used in the image based testing of the set-up. We are also thankful to RRCAT Indore, in particular the Indus synchrotron operation division and health physics unit, for their support during the experiments.

\section{References}

Agrawal, A., Singh, B., Kashyap, Y., Shukla, M. \& Gadkari, S. (2017). J. Radiat. Ca. Res. 8, 153.

Agrawal, A. K., Singh, B., Kashyap, Y. S., Shukla, M., Manjunath, B. S. \& Gadkari, S. C. (2019). J. Synchrotron Rad. 26, 1797-1807.

Agrawal, A. K., Singh, B., Kashyap, Y. S., Shukla, M., Sarkar, P. S. \& Sinha, A. (2015). J. Synchrotron Rad. 22, 1531-1539.

Ay, M. R., Mehranian, A., Maleki, A., Ghadiri, H., Ghafarian, P. \& Zaidi, H. (2013). Phys. Med. 29, 249-260.

Bachche, S., Nonoguchi, M., Kato, K., Kageyama, M., Koike, T., Kuribayashi, M. \& Momose, A. (2017). Sci. Rep. 7, 3-9.

Berujon, S., Wang, H., Alcock, S. \& Sawhney, K. (2014). Opt. Express, 22, 6438.

Chhajed, M., Yadav, C., Agrawal, A. K. \& Maji, P. K. (2019). Carbohydr. Polym. 226, 115286.

Choudhary, A., Pratihar, S. K., Agrawal, A. K. \& Behera, S. K. (2018). Adv. Eng. Mater. 20, 1-7.

Cook, D. N. \& Nakano, H. (2011). Allergens and Respiratory Pollutants, edited by M. A. Williams, pp. 139-156. Cambridge: Woodhead Publishing.

Davison, T. M., Derrick, J. G., Collins, G. S., Bland, P. A., Rutherford, M. E., Chapman, D. J. \& Eakins, D. E. (2017). Proc. Eng. 204, 405412.

Deb, S. K., Singh, G. \& Gupta, P. D. (2013). J. Phys. Conf. Ser. 425, 072009.

Di Michiel, M., Merino, J. M., Fernandez-Carreiras, D., Buslaps, T., Honkimäki, V., Falus, P., Martins, T. \& Svensson, O. (2005). Rev. Sci. Instrum. 76, 1-7.

Douissard, P. A., Cecilia, A., Rochet, X., Chapel, X., Martin, T., Kamp, T., Helfen, L., Baumbach, T., Luquot, L., Xiao, X., Meinhardt, J. \& Rack, A. (2012). J. Instrum. 7, P09016.

Fatima, A., Kataria, S., Baghel, L., Guruprasad, K. N., Agrawal, A. K., Singh, B., Sarkar, P. S., Shripathi, T. \& Kashyap, Y. (2017). J. Synchrotron Rad. 24, 232-239.

García-Moreno, F., Kamm, P. H., Neu, T. R. \& Banhart, J. (2018). J. Synchrotron Rad. 25, 1505-1508.

Huang, J. Y., Jin, K. S., Lim, J. H., Kim, H. Y., Jang, S. D., Choi, H. J., Gil, K. H. \& Lee, S. K. (2010). J. Korean Phy. Soc. 56, 2077-2082.
Jung, H., Kim, H. J., Hong, S., Hong, J. O., Jeong, H. K., Je, J. H., Kim, B. R. \& Yoo, H. S. (2002). IEEE Trans. Nucl. Sci. 49, 2262-2267.

Lai, B., Yun, W., Xiao, Y., Yang, L., Legnini, D., Cai, Z., Krasnoperova, A., Cerrina, F., DiFabrizio, E., Grella, L. \& Gentili, M. (1995). Rev. Sci. Instrum. 66, 2287-2289.

Manzoor, M. A. P., Agrawal, A. K., Singh, B., Mujeeburahiman, M. \& Rekha, P. D. (2019). PLoS One, 14, e0214003.

Mishra, A., Melo, J. S., Agrawal, A., Kashyap, Y. \& Sen, D. (2020). Colloids Surf. B Biointerfaces, 188, 110796.

Mittone, A., Fardin, L., Di Lillo, F., Fratini, M., Requardt, H., Mauro, A., Homs-Regojo, R. A., Douissard, P.-A., Barbone, G. E., Stroebel, J., Romano, M., Massimi, L., Begani-Provinciali, G., Palermo, F., Bayat, S., Cedola, A., Coan, P. \& Bravin, A. (2020). J. Synchrotron Rad. 27, 1347-1357.

Patterson, B. M., Cordes, N. L., Henderson, K., Xiao, X. \& Chawla, N. (2018). Microsc. Microanal. 24, 1002-1003.

Rack, A., García-Moreno, F., Helfen, L., Mukherjee, M., Jiménez, C., Rack, T., Cloetens, P. \& Banhart, J. (2013). Appl. Opt. 52, 81228127.

Rack, A., Garcia-Moreno, F., Schmitt, C., Betz, O., Cecilia, A., Ershov, A., Rack, T., Banhart, J. \& Zabler, S. (2010). J. Xray Sci. Technol. 18, 429-441.

Rack, A., Weitkamp, T., Bauer Trabelsi, S., Modregger, P., Cecilia, A., dos Santos Rolo, T., Rack, T., Haas, D., Simon, R., Heldele, R., Schulz, M., Mayzel, B., Danilewsky, A. N., Waterstradt, T., Diete, W., Riesemeier, H., Müller, B. R. \& Baumbach, T. (2009). Nucl. Instrum. Methods B, 267, 1978-1988.

Rana, N., Rawat, D., Parmar, M., Dhawan, D. K., Bhati, A. K. \& Mittal, B. R. (2015). J. Med. Phys. 40, 198-206.

Reinhart, G., Nguyen-Thi, H., Mangelinck-Noël, N., Baruchel, J. \& Billia, B. (2014). JOM, 66, 1408-1414.

Rio, M. S. del \& Dejus, R. J. (2011). Proc. SPIE, 8141, 368-372.

Rivers, M. L. (2016). Proc. SPIE, 9967, 99670X.

Sena, G., Almeida, A. P., Braz, D., Nogueira, L. P., Soares, J., Azambuja, P., Gonzalez, M. S., Tromba, G. \& Barroso, R. C. (2015). Radiat. Phys. Chem. 115, 179-182.

Singh, B., Agrawal, A. K., Kashyap, Y. S. \& Gadkari, S. C. (2017). AIP Conf. Proc. 1832, 060010.

Singh, S. S., Williams, J. J., Hruby, P., Xiao, X., De Carlo, F. \& Chawla, N. (2014). Integrating Mater. 3, 109-122.

Tan, D., Lee, T. L., Khong, J. C., Connolley, T., Fezzaa, K. \& Mi, J. (2015). Metall. Mater. Trans. A, 46, 2851-2861.

Thomas, A., Johnson, E., Agrawal, A. K. \& Bera, J. (2019). J. Mater. Res. 34, 3798-3809.

Wang, Y., De Carlo, F., Mancini, D. C., McNulty, I., Tieman, B., Bresnahan, J., Foster, I., Insley, J., Lane, P., von Laszewski, G., Kesselman, C., Su, M. \& Thiebaux, M. (2001). Rev. Sci. Instrum. 72, 2062-2068.

Wysokinski, T. W., Chapman, D., Adams, G., Renier, M., Suortti, P. \& Thomlinson, W. (2007). Nucl. Instrum. Methods Phys. Res. A, 582, 73-76.

Yadav, R., Agrawal, A. K., Ali, M., Kumar, A., Singh, B., Kashyap, Y., Sinha, A., Gadkari, S. C. \& Pandey, B. N. (2018). Toxicol. Environ. Heal. Sci. 10, 97-106.

Yang, B., Johnson, D. A. \& Shim, S. H. (1993). Corrosion, 49, 499-513. Yashiro, W., Ueda, R., Kajiwara, K., Noda, D. \& Kudo, H. (2017). Jpn. J. Appl. Phys. 56, 112503.

Zhou, T., Wang, H., Connolley, T., Scott, S., Baker, N. \& Sawhney, K. (2018). J. Synchrotron Rad. 25, 801-807. 